\title{
Discrepancies between selected Pareto optimal plans and final deliverable plans in radiotherapy multi-criteria optimization
}

Archonteia Kyroudi $^{a}$, Kristoffer Petersson ${ }^{a}$, Sarah Ghandour ${ }^{b}$, Marc Pachoud ${ }^{b}$, Oscar Matzinger ${ }^{b}$, Mahmut Ozsahin ${ }^{c}$, Jean Bourhis ${ }^{c}$, François Bochud ${ }^{\mathrm{a}}$, Raphaël Moecklia

a Institute of Radiation Physics (IRA), Lausanne University Hospital, Lausanne, Switzerland
b Department of Radiation Oncology, Hôpital Riviera-Chablais, Vevey, Switzerland
${ }^{c}$ Department of Radiation Oncology, Lausanne University Hospital, Lausanne, Switzerland

(C) 2016. This manuscript version is made available under the Elsevier user license http://www.elsevier.com/open-access/userlicense/1.0/ 
Abstract

Multi-criteria optimization provides decision makers with a range of clinical choices through Pareto plans that can be explored during real time navigation and then converted into deliverable plans. Our study shows that dosimetric differences can arise between the two steps, which could compromise the clinical choices made during navigation.

Keywords: Multi-criteria optimization, Pareto fronts, navigated, deliverable

Navigation based multi-criteria optimization (MCO) methods have been recently introduced into radiotherapy to support decision makers in finding the most preferred plan among a wide range of mathematically optimal alternatives [1]. An interactive interface allows the decision maker to explore these options during real-time navigation. However, in order to ensure efficient navigation, the navigated plans are near-optimal in the fluence space, where physical machine parameters are not considered. The final deliverable dose is calculated with a direct machine parameter optimization (DMPO) dose algorithm which takes multi-leaf collimator (MLC) segments into account [2].

The choice of optimization method directly impacts the decision making strategies in clinical practice. For conventional inverse treatment planning and evaluation, the decision makers evaluate the quality of a single plan without any evidence to guarantee its optimality or any information regarding the full solution space. In contrast, with MCO decision makers can quickly and efficiently investigate the full range of alternative choices and select a plan that best reflect their clinical preferences [3]. The selection is made among several near-Pareto optimal plans, for which no objective can be improved without deteriorating another. The interactive exploration of trade-offs has been shown to result in different clinical decisions [4], to reduce planning time and to improve plan quality [5]. However, accurate trade-off information is necessary, i.e. a good agreement between navigated and deliverable plans, in order to ensure informed clinical decision making.

The purpose of this study was to present a range of available treatment solutions generated with MCO and to evaluate the dosimetric differences between navigated and deliverable plans. Our aim was to illustrate the implications of the MCO approach to the decision making process and to present the limitations of the two-step method.

\section{Materials and methods}

Five localized prostate cancer cases (seminal vesicles not included) and five non-small cell lung cancer (NSCLC) cases were randomly selected from the patient database of our department for this retrospective planning study. All the critical structures were delineated by the same radiation oncologist. The overlapped volumes between the PTV and the rectum varied for the five prostate cases. The ratio of the overlapped volume to the delineated rectum volume was $13 \%, 25 \%, 9.5 \%, 4.3 \%$ and $10.9 \%$. Lung tumor size and location varied for the five lung cancer cases (Table 1 in the supplementary material). The prescribed dose to the PTV was 78 Gy in 39 fractions (mean dose) for the prostate cases and 60 Gy or 54 Gy in 3, 5 or 8 fractions for the lung cases.

All plans were obtained using the volumetric modulated arc therapy (VMAT) multi-criteria optimization technique in the RayStation ${ }^{\circledR}$ (RaySearch Laboratories AB, Stockholm, Sweden) treatment planning system version 4.0, modeling an Elekta Versa HD ${ }^{\mathrm{TM}}$ Linear Accelerator (Elekta AB, Stockholm, Sweden) with the Agility ${ }^{\mathrm{TM}}$ MLC. For each optimization problem two objectives were chosen representing the main conflicting criteria during optimization (prostate: rectum sparing versus PTV under-dosage; lung: healthy lung sparing versus PTV under-dosage). In order to reduce the multi-dimensionality of the problem, optimization constraints were applied to all the organs at risk (OARs) not involved in the main trade-off (Table 2 in the supplementary material). Similar objectives and constraints were used for all cases to set up the treatment planning problem in the MCO module. However, there were numerical differences in the objectives and constraint values between cases due to anatomical differences between patients. All cases were calculated for a single arc VMAT treatment.

The set of navigated and deliverable plans were visualized as Pareto fronts, representing the trade-off under evaluation. PTV underdosage was evaluated through the "[100- $\left.\mathrm{V}_{95 \%}\right]$ relative volume", where $\mathrm{V}_{95 \%}$ is the volume receiving at least $95 \%$ of the prescription dose. Rectum sparing was evaluated through the $D_{50 \%}$ evaluation parameter, where $D_{50 \%}$ is the dose received by $50 \%$ of the rectum volume [6]. For the lung cases, $V_{5 G y}$ was chosen as the evaluation parameter for healthy lung tissue (lung-PTV) [7]. 


\section{Results and discussion}

In two out of the five prostate cases (cases 4 and 5, see Figure 1 in the supplementary material) there was a clear improvement for the deliverable plans with better PTV coverage and better rectum sparing. For the other cases the disagreement proved more random, resulting in plans of better or worse final quality concerning the trade-off evaluated. For all cases, full PTV coverage could not be achieved for the deliverable plans (Figure 1, and Figure 1 in the supplementary material). The maximum improvement of PTV coverage in prostate cases for a final deliverable plan compared to a navigated one was $2.9 \%$, while the maximum deterioration was $3.4 \%$. For the rectum sparing evaluation parameter, the maximum improvement was $3.3 \mathrm{~Gy}$ and the maximum deterioration was 1.7 Gy. Similar results have been demonstrated for a pancreas, a prostate and a brain case by Craft et al. (2008) [8]. Those authors suggested that the two-step methodology does not deteriorate the plan quality and can result in improved normal tissue sparing after segmentation. However, in our results this improvement was achieved partly by compromising other parameters, such as increasing doses to other OARs or by creating small "hotspots" (not visible in our two-dimensional Pareto fronts).

For the lung cases, the final deliverable plans were systematically worse than the navigated ones (Figure 1, and Figure 2 in the supplementary material). Significant deterioration of up to $17.1 \%$ existed for the PTV under-dosage parameter in three of the five cases (cases 2, 3, and 5). The largest discrepancy between navigated and deliverable fronts occurred in the plans with the smallest PTV $\left(5.2 \mathrm{~cm}^{3}\right.$ and $\left.9.6 \mathrm{~cm}^{3}\right)$. The maximum improvement for PTV coverage between the navigated and final deliverable plan was 2.3\%. The lung-PTV evaluation parameter showed no improvement in any of the five lung cases for the deliverable plans compared to the navigated ones (Figure 1, and Figure 2 in the supplementary material). The maximum deterioration was $3.9 \%$.

Plan deterioration for the lung cases might be expected since dose calculation in fluence-based treatment plans does not take into account the effect of lateral electron transport in the presence of heterogeneities. Dosimetric differences for lung cases calculated with correction-based compared to collapsed cone convolution algorithms are well documented in the literature [9]. In our study, the degree of discrepancy between navigated and deliverable plans varied for the five lung cases and appeared to be related to the PTV volume size. In the two cases with a PTV volume smaller than $10 \mathrm{~cm}^{3}$, PTV coverage was dramatically decreased (up to $17 \%$ additional under-coverage), leading to unacceptable plans. Due to our limited number of cases, we cannot prove a correlation between tumor size and PTV under-dosage. However, a study by van der Voort van Zyp et al. (2010) reported larger differences on $\mathrm{D}_{95 \%}$ for smaller tumors between different calculation algorithms [10]. Additionally, Akino et al. (2014) reported a logarithmic relationship between the PTV volume and the effect of lateral electron transport in dose calculations for stereotactic body radiotherapy (SBRT) [11]. Comparisons with a highly accurate algorithm (e.g. based on Monte Carlo calculations), could evaluate the accuracy of both the fluence-based dose calculation algorithm used to get the navigated plans and the convolution/superposition algorithm used to get the deliverable plans [12].

Our results show that, in terms of the evaluation parameters selected, a wide range of near-Pareto optimal plans are available during navigation. This is particularly the case for the prostate cases where the $\mathrm{D}_{50 \%}$ rectum evaluation parameter spans a dosimetric range from 25 Gy (case 5) up to 46 Gy (case 3). Within the clinically acceptable PTV under-dosage (0-5\%), the decision maker can select either of the two extreme plans $(0 \%$ or $5 \%$ under-dosage) which are mathematically equivalent. However, the first choice would result, for example in case 3, in 46 additional Gy to the $D_{50 \%}$ to the rectum. It is clear that the MCO approach changes the task at hand to the decision maker, which is no longer only an inference task of judging the plan quality but a preferential one.

The lack of systematic differences between navigated and deliverable plans makes it difficult to predict the dosimetric change, its direction and its magnitude (Figure 1, and Figures 1 and 2 in the supplementary material). If the navigated plan is poorly correlated to the deliverable one, the level of uncertainty during the decision making is increased and the whole process becomes less reliable. Furthermore, the benefit of presenting trade-off information is lost if the trade-off characteristics that supported a clinical choice cannot be reproduced in the final deliverable plan. To our knowledge, the latest version of RayStation has addressed this problem for IMRT plans by making all the plans in the Pareto database deliverable [13]. However, the problem has not been addressed for VMAT plans, for which the challenges are greater due to the larger number of degrees of freedom that is available to shape the dose distribution.

The methodology of reducing the total treatment plan problem to a bi-criteria problem allowed us to efficiently visualize the selected trade-off in two dimensional Pareto fronts. However, the selected problem formulation differs from the optimization formulation in everyday clinical practice. With a full objective problem formulation and less constraints, better values for the other OARs could probably be achieved. On the other hand, Craft et al. (2005) suggested that the choice of objective function for normal tissue does not greatly affect the plan database, particularly for small organ sizes where maximum and mean doses are correlated [14]. Therefore, we consider that the number of constraints mainly shaped the solution space and that the choice of the objective functions had little effect on the trade-offs.

By taking into account the multi-dimensionality of the problem, MCO offers the advantage of a full choice of near-Pareto optimal plans, it reveals all the trade-offs involved and it conveys information to support informed decision making. With navigation in the fluence space the decision maker benefits from valuable trade-off information rapidly and without solving the computationally expensive final deliverable plan problem. As a result, it suggests a paradigm shift in the decision making process during which the 
decision maker gets an insight of the solution space by navigating through alternative compromises. However, dosimetric disagreements due to the two-step methodology may limit the benefit of "fine-tuning" the optimal plan according to clinical preferences. Especially for cases with small targets surrounded by low density tissues, the final deliverable plan does not necessarily reflect the clinical preferences that motivated a choice during navigation.

\section{Acknowledgements}

The project was supported by the Swiss National Science Foundation (FNS Project 320030_149489/1). 

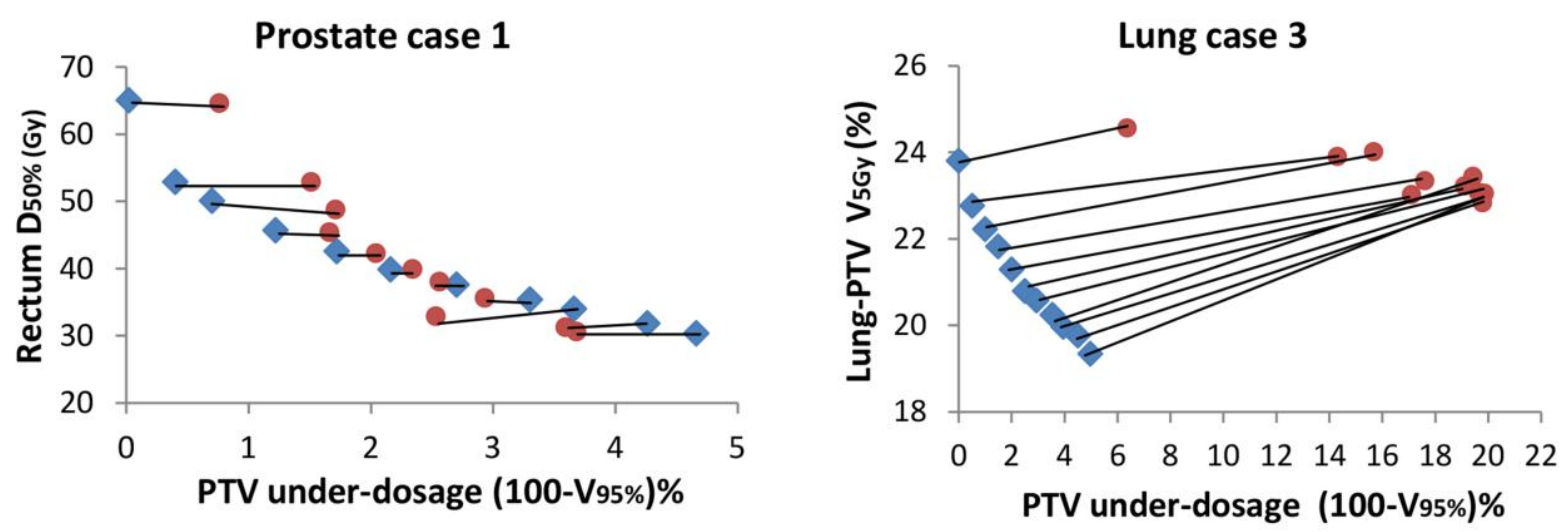

Figure 1: Pareto front representation of the trade-off "rectum sparing versus PTV under-dosage" for a prostate cancer patient (left) and the trade-off "lung-PTV sparing versus PTV under-dosage" for a lung cancer patient (right). Diamonds represent navigated Pareto plans and circles represent deliverable plans. The lines indicate the navigated solutions leading to the corresponding deliverable plans.

\section{REFERENCES}

[1] Küfer K-H, Scherrer A, Monz M, et al. Intensity-modulated radiotherapy - a large scale multi-criteria programming problem. OR Spectrum. 2003;25:223-49.

[2] Hårdemark B, Liander A, Rehbinder H, Löf J. Direct machine parameter optimization with RayMachine in Pinnacle. RaySearch White Paper. 2003.

[3] Craft D, Halabi T, Shih HA, Bortfeld T. An approach for practical multiobjective IMRT treatment planning. Int J Radiat Oncol Biol Phys. 2007;69:1600-7.

[4] Hong TS, Craft DL, Carlsson F, Bortfeld TR. Multicriteria optimization in intensity-modulated radiation therapy treatment planning for locally advanced cancer of the pancreatic head. Int J Radiat Oncol Biol Phys. 2008;72:1208-14.

[5] Craft DL, Hong TS, Shih HA, Bortfeld TR. Improved planning time and plan quality through multicriteria optimization for intensity-modulated radiotherapy. Int J Radiat Oncol Biol Phys. 2012;82:e83-90.

[6] Peeters ST, Heemsbergen WD, Koper PC, et al. Dose-response in radiotherapy for localized prostate cancer: results of the Dutch multicenter randomized phase III trial comparing $68 \mathrm{~Gy}$ of radiotherapy with 78 Gy. J Clin Oncol. 2006;24:1990-6.

[7] Yorke ED, Jackson A, Rosenzweig KE, et al. Correlation of dosimetric factors and radiation pneumonitis for non-small-cell lung cancer patients in a recently completed dose escalation study. Int J Radiat Oncol Biol Phys. 2005;63:672-82.

[8] Craft D, Carlsson F, Bortfeld T, Rehbinder H. SU-GG-T-527: Multi-Objective IMRT Planning Which Produces Deliverable Plans. Med Phys. 2008;35:2846.

[9] Carrasco P, Jornet N, Duch MA, et al. Comparison of dose calculation algorithms in phantoms with lung equivalent heterogeneities under conditions of lateral electronic disequilibrium. Med Phys. 2004;31:2899-911. 
[10] van der Voort van Zyp NC, Hoogeman MS, van de Water S, et al. Clinical introduction of Monte Carlo treatment planning: a different prescription dose for non-small cell lung cancer according to tumor location and size. Radiother Oncol. 2010;96:55-60.

[11] Akino Y, Das IJ, Cardenes HR, Desrosiers CM. Correlation between target volume and electron transport effects affecting heterogeneity corrections in stereotactic body radiotherapy for lung cancer. J Radiat Res. 2014;55:754-60.

[12] Ottosson RO, Karlsson A, Behrens CF. Pareto front analysis of 6 and 15 MV dynamic IMRT for lung cancer using pencil beam, AAA and Monte Carlo. Phys Med Biol. 2010;55:4521-33.

[13] Fredriksson A, Bokrantz R. Deliverable navigation for multicriteria IMRT treatment planning by combining shared and individual apertures. Phys Med Biol. 2013;58:7683-97.

[14] Craft D, Halabi T, Bortfeld T. Exploration of tradeoffs in intensity-modulated radiotherapy. Phys Med Biol. 2005;50:5857-68. 